\title{
An Improvement in the Selection Process of Machine Translation Using Inductive Learning with Genetic Algorithms
}

\author{
Hiroshi Echizen-ya \\ Kenji Araki \\ Division of Electronics and Information Dept. of Electronics and Information \\ Hokkaido University \\ Sapporo, 060 Japan \\ echi@hudk.hokudai.ac.jp \\ Hokkai-Gakuen University \\ Sapporo, 064 Japan \\ araki@eli.hokkai-s-u.ac.jp \\ Yoshikazu Miyanaga Koji Tochinai \\ Division of Electronics and Information \\ Hokkaido University Sapporo, 060 Japan \\ \{miyanaga, tochinai\}@hudk.hokudai.ac.jp
}

\begin{abstract}
We proposed a method of machine translation using inductive learning with genetic algorithms, and confirmed the effectiveness of applying genetic algorithms. However, the system based on this method produces many erroneous translation rules that cannot be completely removed from the dictionary. Therefore, we need to improve how to apply genetic algorithms to be able to remove erroneous translation rules from the dictionary. In this paper, we describe this improvement in the selection process and the results of evaluation experiments.
\end{abstract}

\section{Introduction}

Many studies have been carried out on machine translation and a number of problems has been recognized. Rule-based machine translation (Hutchins and Somers, 1992) could not deal adequately with various linguistic phenomena due to the use of limited rules. To resolve this problem, Example-based machine translation (Sato and Nagao, 1990) has recently been proposed. However, this method requires many translation examples to achieve a practical and high-quality translation.

Echizen-ya and others previously proposed a method of Machine Translation using Inductive Learning with Genetic Algorithms (GA-ILMT), and this method has been evaluated(Echizen-ya et al., 1996). By applying genetic algorithms, we consider that our proposed method can effectively solve problems that Example-based machine translation would require many translation examples. However, the results of the evaluation experiments show that this method has some problems. The main problem is that many erroneous translation rules are produced and these rules cannot be completely removed from the dictionary. Therefore, we need to improve how to apply genetic algorithms to be able to remove erroneous translation rules. In this paper, we describe an improvement in the selection process of GA-ILMT, and confirm the effectiveness of improvement in the selection process of GA-ILMT.

\section{Outline of Translation Method}

Figure 1 shows the outline of our proposed translation method. First, the user inputs a source sentence in English. Second, in the translation process, the system produces several candidates of translation results using translation rules extracted in the learning process. Third, the user proofreads the translated sentences if they include some errors. Fourth, in the feedback process, the system determines the fitness value of translation rules used in the translation process and performs the selection process of erroneous translation rules. In the learning process, new translation examples are automatically produced by crossover and mutation, and various translation rules are extracted from the translation examples by inductive learning.

\section{Improvement in Selection Process}

In the previous method of selection process described in Section 2, translation rules are evaluated only when they are used in the translation process. These translation rules are part of all the translation rules in the dictionary. Therefore, many erroneous 


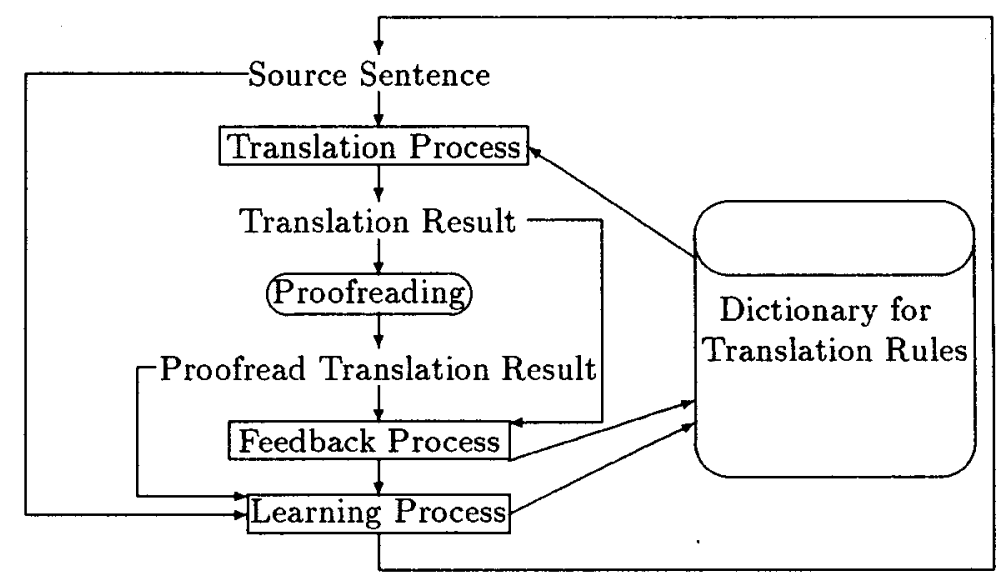

Figure 1: Outline of the translation method

translation rules cannot be completely removed from the dictionary.

To resolve this problem, we propose an improvement in the selection process. Our proposed improvement does not require any analytical knowledge as initial condition. Methods that use analytical knowledge have some problems, such as difficulty in dealing with unregistered words. We consider that this problem can be resolved by the learning method without any analytical knowledge. Therefore, we consider that our proposed improvement can remove many erroneous translation rules by utilizing only the given translation examples without the requirement of analytical knowledge.

The system evaluates the translation rules by utilizing the given translation examples directly. Namely, it determines whether a combination of the English word and the Japanese word in a translation rule is true or false by utilizing the given translation examples. The combination may be true when it exists in a given translation example. For example, the combination of words which are "I" in English and "Watashi (In Japanese "I")" in Japanese is true when this combination exists in a given translation example. On the other hand, the combination of words which are "volleyball" in English and "Basukettoboru(In Japanese "basketball")" in Japanese is false when this combination does not exist in all given translation examples. In the all combinations of words in a translation rule, the system determines whether the each combination of words is true or false. And the system determines the rate of error based on the number of erroneous combinations, and removes the translation rules for which the rate of error is high.

\section{Experiments}

In the experiments, 461 translation examples were used as data. The examples were taken from a textbook (Hasegawa et al., 1991) for first-grade junior

\footnotetext{
${ }^{1}$ Italic means pronunciation of Japanese
}

high school students. All of the translation examples were processed by the method outlined in Figure 1 . The initial dictionary was empty. The experiments were carried out with and without the improvement for the selection process described in Section 3. In the experiments, the precision increased from $87.5 \%$ to $93.7 \%$ and the recall increased from $4.5 \%$ to $56.0 \%$.

\section{Conclusion}

In the previous selection process, the translation rules are evaluated only when they are used in the translation process. Therefore, the translation rules which are not used in the translation process are never removed from the dictionary. However, the proposed improvement can evaluate all of the produced translation rules by utilizing only the given translation examples.

\section{Acknowledgements}

The part of this research has been done during the second author Kenji Araki's stay at CSLI of Stanford University. We would like to express our special appreciation to the stuffs of CSLI.

\section{References}

W. John Hutchins and Harold L. Somers. 1992. An Introduction to Machine Translation. $A C A$ DEMIC PRESS. (London)

Sato, S. and Nagao, M. 1990. Toward Memorybased Translation. In Proceedings of the Coling'90, pages 247-252, Helsinki, Finland, August.

Echizen-ya, H., Araki, K., Momouchi, Y. and Tochinai, K. 1996. Machine Translation Method Using Inductive Learning with Genetic Algorithms. In Proceedings of the Coling'96, pages 1020-1023, Copenhagen, Denmark, August.

Hasegawa, K. et al., 1991. One World English Course 1 New Edition. Kyoiku Shuppan. (Tokyo). 